\title{
SUATU KAJIAN TENTANG HIMPUNAN LUNAK KABUR (FUZZY SOFT SET) DAN APLIKASINYA
}

\author{
PRIMA PUTRI ADHA UTAMI \\ Program Studi Matematika, \\ Fakultas Matematika dan Ilmu Pengetahuan Alam, Universitas Andalas, \\ Kampus UNAND Limau Manis Padang, Indonesia, \\ email : primaputriadhautami@gmail.com
}

\begin{abstract}
Abstrak. Dalam paper ini dikaji tentang teori himpunan lunak kabur beserta operasioperasinya. Kemudian didefinisikan operator fuzzy soft aggregation yang dapat diterapkan dalam proses pengambilan keputusan. Terakhir diberikan contoh yang menunjukkan bahwa metode ini dapat diaplikasikan pada masalah yang mengandung ketidaktentuan. Paper ini mengkaji kembali referensi [2].

Kata Kunci: Himpunan lunak, himpunan lunak kabur, fungsi keanggotaan, ketidaktentuan
\end{abstract}

\section{Pendahuluan}

Misal diberikan himpunan $U$ yang disebut himpunan universal atau semesta, dan $E$ himpunan dari parameter, $P(U)$ adalah power set dari $U$, dan $A \subseteq E$.

Definisi 1.1. [2] Suatu soft set $F_{A}$ atas $U$ adalah himpunan yang didefinisikan oleh fungsi $f_{A}$

$$
f_{A}: E \rightarrow P(U)
$$

sedemikian sehingga

$$
f_{A}(x)=\emptyset \text { jika } x \notin A .
$$

Fungsi $f_{A}$ dikatakan fungsi aproksimasi dari soft set $F_{A}$, dan nilai dari $f_{A}(x)$ disebut $x$-elemen dari soft set untuk semua $x \in E$. Soft set atas $U$ dapat dituliskan dalam himpunan pasangan terurut

$$
F_{A}=\left\{\left(x, f_{A}(x)\right): x \in E, f_{A}(x) \in P(U)\right\} .
$$

Himpunan semua soft set atas $U$ dinotasikan dengan $S(U)$.

Definisi 1.2. [2] Misal $U$ adalah himpunan universal. Himpunan fuzzy $X$ atas $U$ adalah himpunan yang didefinisikan oleh fungsi

$$
\mu_{X}: U \rightarrow[0,1],
$$

dimana $\mu_{X}$ disebut fungsi keanggotaan dari $X$, dan nilai dari $\mu_{X}$ disebut nilai dari keanggotaan untuk $u \in U$. Nilai tersebut menunjukkan derajat dari u pada 
himpunan fuzzy $X$. Himpunan fuzzy $X$ atas $U$ dapat dituliskan sebagai himpunan pasangan terurut

$$
X=\left\{\left(\mu_{X}(u) / u\right): u \in U, \mu_{X}(u) \in[0,1]\right\} .
$$

Koleksi dari himpunan-himpunan fuzzy atas $U$ dinotasikan dengan $F(U)$.

Definisi 1.3. [3] Misal $A$ dan $B$ adalah dua himpunan fuzzy atas $U$. Maka $A$ adalah himpunan bagian dari $B$ dinotasikan dengan $A \subseteq B$ jika dan hanya jika $\mu_{A}(x) \leq \mu_{B}(x)$ untuk setiap $x \in U$.

Definisi 1.4. [3] Misal $A, B \in F(U)$. Maka, $A=B$ jika dan hanya jika $A \subseteq B$ dan $B \subseteq A$.

Definisi 1.5. [3] Misal $A \in F(U)$. Maka, komplemen dari A adalah

$$
A^{c}=\left\{\left(x, \mu_{A^{c}}(x)\right): x \in U \text { dan } \mu_{A^{c}}(x)=1-\mu_{A}(x)\right\} .
$$

Definisi 1.6. [3] Misal $A, B \in F(U)$. Maka, gabungan dari $A$ dan $B$ yang dinotasikan dengan $A \cup B$ didefinisikan sebagai

$$
\mu_{A \cup B}(x)=\max \left\{\mu_{A}(x), \mu_{B}(x)\right\}
$$

untuk setiap $x \in U$ dan $\mu_{A \cup B}(x) \in[0,1]$.

Definisi 1.7. [3] Misal $A, B \in F(U)$. Maka, irisan dari $A$ dan $B$ yang dinotasikan dengan $A \cap B$ didefinisikan sebagai

$$
\mu_{A \cap B}(x)=\min \left\{\mu_{A}(x), \mu_{B}(x)\right\}
$$

untuk setiap $x \in U$ dan $\mu_{A \cap B}(x) \in[0,1]$.

\section{Himpunan Lembut Kabur (Fuzzy Soft Set)}

Notasikan $\Gamma_{A}, \Gamma_{B}, \Gamma_{C}, \cdots$ untuk menyatakan fuzzy soft set dan $\gamma_{A}, \gamma_{B}, \gamma_{C}, \cdots$ untuk fungsi aproksimasi fuzzy.

Definisi 2.1. [2] Suatu $f$ s-set atas $U$ adalah himpunan yang didefinisikan oleh fungsi $\gamma_{A}$

$$
\gamma_{A}: E \rightarrow F(U) \text { sedemikian sehingga } \gamma_{A}(x)=\emptyset \text { jika } x \notin A \text {. }
$$

Fungsi $\gamma_{A}$ disebut fungsi aproksimasi fuzzy dari fuzzy soft set $\Gamma_{A}$, dan nilai $\gamma_{A}(x)$ disebut $x$-elemen dari fuzzy soft set untuk semua $x \in E$. fuzzy soft set $\Gamma_{A}$ atas $U$ dapat dituliskan dengan himpunan pasangan terurut

$$
\Gamma_{A}=\left\{\left(x, \gamma_{A}(x)\right): x \in E, \gamma_{A}(x) \in F(U)\right\} \text {. }
$$

Himpunan dari semua $f$ s-sets atas $U$ dinotasikan dengan $F S(U)$.

Definisi 2.2. [2] Misal $\Gamma_{A} \in F S(U)$. Jika $\gamma_{A}(x)=\emptyset$ untuk setiap $x \in E$, maka $\Gamma_{A}$ disebut empty $f$ s-set, dinotasikan dengan $\Gamma_{\Phi}$. 
Definisi 2.3. [2] Misal $\Gamma_{A} \in F S(U)$. Jika $\gamma_{A}(x)=U$ untuk setiap $x \in A$, maka $\Gamma_{A}$ disebut $A$-universal $f$ s-set, dinotasikan dengan $\Gamma_{\bar{A}}$. Jika $A=E$, maka $\Gamma_{A}$ disebut universal $f$ s-set, dinotasikan dengan $\Gamma_{\bar{E}}$.

Definisi 2.4. [2] Misal $\Gamma_{A}, \Gamma_{B} \in F S(U)$. Maka $\Gamma_{A}$ adalah fs-subset dari $\Gamma_{B}$, dinotasikan dengan $\Gamma_{A} \tilde{\subseteq} \Gamma_{B}$, jika $\gamma_{A}(x) \subseteq \gamma_{B}(x)$ untuk setiap $x \in E$.

Proposisi 2.5. [2] Misal $\Gamma_{A}, \Gamma_{B} \in F S(U)$. Maka,

(1) $\Gamma_{A} \tilde{\widetilde{\subseteq}} \Gamma_{\bar{E}}$.

(2) $\Gamma_{\Phi} \tilde{\simeq} \Gamma_{A}$.

(3) $\Gamma_{A} \tilde{\simeq} \Gamma_{A}$.

(4) $\Gamma_{A} \tilde{\simeq} \Gamma_{B}$ dan $\Gamma_{B} \tilde{\tilde{\subseteq}} \Gamma_{C} \Rightarrow \Gamma_{A} \tilde{\simeq} \Gamma_{C}$.

Bukti. Dapat dibuktikan dengan menggunakan Definisi 1.3.

Definisi 2.6. [2] Misal $\Gamma_{A}, \Gamma_{B} \in F S(U)$. Maka, $\Gamma_{A}=\Gamma_{B}$ jika dan hanya jika $\gamma_{A}(x)=\gamma_{B}(x)$ untuk setiap $x \in E$.

Proposisi 2.7. [2] Misal $\Gamma_{A}, \Gamma_{B}, \Gamma_{C} \in F S(U)$. Maka,

(1) Jika $\Gamma_{A}=\Gamma_{B}$ dan $\Gamma_{B}=\Gamma_{C}, \operatorname{maka} \Gamma_{A}=\Gamma_{C}$.

(2) $\Gamma_{A} \tilde{\simeq} \Gamma_{B}$ dan $\Gamma_{B} \tilde{\subseteq} \Gamma_{A}$ jika dan hanya jika $\Gamma_{A}=\Gamma_{B}$.

Bukti. Misal $\Gamma_{A}, \Gamma_{B}, \Gamma_{C} \in F S(U)$. Menggunakan Definisi 1.4 akan dibuktikan

(1) $\Gamma_{A}=\Gamma_{B}$ berarti $\mu_{\gamma_{A}(x)}(u)=\mu_{\gamma_{B}(x)}(u)$ untuk setiap $x \in E$ dan $u \in U$. $\Gamma_{B}=\Gamma_{C}$ berarti $\mu_{\gamma_{B}(x)}(u)=\mu_{\gamma_{C}(x)}(u)$ untuk setiap $x \in E$ dan $u \in U$. $\mu_{\gamma_{A}(x)}(u)=\mu_{\gamma_{B}(x)}(u)$ dan $\mu_{\gamma_{B}(x)}(u)=\mu_{C}(x)$ berarti $\mu_{\gamma_{A}(x)}(u)=\mu_{\gamma_{C}(x)}(u)$.

(2) $(\Rightarrow)$

Karena $\mu_{\gamma_{A}(x)}(u) \leqslant \mu_{\gamma_{B}(x)}(u)$ dan $\mu_{\gamma_{B}(x)}(u) \leqslant \mu_{\gamma_{A}(x)}(u)$, maka $\mu_{\gamma_{A}(x)}(u)=$ $\mu_{\gamma_{B}(x)}(u)$ untuk setiap $x \in E$ dan $u \in U$.

$(\Leftarrow)$

Dan karena $\mu_{\gamma_{A}(x)}(u)=\mu_{\gamma_{B}(x)}(u)$ berarti $\mu_{\gamma_{A}(x)}(u) \leqslant \mu_{\gamma_{B}(x)}(u)$ dan $\mu_{\gamma_{B}(x)}(u) \leqslant \mu_{\gamma_{A}(x)}(u)$.

Definisi 2.8. [2] Misal $\Gamma_{A} \in F S(U)$. Maka, komplemen $\Gamma_{A}^{\bar{c}}$ dari $\Gamma_{A}$ adalah suatu fs-set sedemikian sehingga

$$
\gamma_{A^{\bar{c}}}(x)=\gamma_{A}^{c}(x) \text {, untuk setiap } x \in E,
$$

dimana $\gamma_{A}^{c}(x)$ adalah komplemen dari himpunan $\gamma_{A}(x)$.

Proposisi 2.9. [2] Misal $\Gamma_{A} \in F S(U)$. Maka,

(1) $\left(\Gamma_{A}^{\bar{c}}\right)^{\bar{c}}=\Gamma_{A}$

(2) $\Gamma_{\Phi}^{\bar{c}}=\Gamma_{\bar{E}}$.

Bukti. Proposisi ini dapat dibuktikan dengan menggunakan Definisi 1.5. 
Definisi 2.10. [2] Misal $\Gamma_{A}, \Gamma_{B} \in F S(U)$. Maka, gabungan dari $\Gamma_{A}$ dan $\Gamma_{B}$, dinotasikan dengan $\Gamma_{A} \tilde{\cup} \Gamma_{B}$, didefinisikan oleh fungsi aproksimasi fuzzy

$$
\gamma_{A \tilde{\cup} B}(x)=\gamma_{A}(x) \cup \gamma_{B}(x) \text { untuk setiap } x \in E \text {. }
$$

Proposisi 2.11. [2] Misal $\Gamma_{A}, \Gamma_{B}, \Gamma_{C} \in F S(U)$. Maka,

(1) $\Gamma_{A} \tilde{\cup} \Gamma_{A}=\Gamma_{A}$.

(2) $\Gamma_{A} \tilde{\cup} \Gamma_{\Phi}=\Gamma_{A}$.

(3) $\Gamma_{A} \tilde{\cup} \Gamma_{\bar{E}}=\Gamma_{\bar{E}}$.

(4) $\Gamma_{A} \tilde{\cup} \Gamma_{B}=\Gamma_{B} \tilde{\cup} \Gamma_{A}$.

(5) $\left(\Gamma_{A} \tilde{\cup} \Gamma_{B}\right) \tilde{\cup} \Gamma_{C}=\Gamma_{A} \tilde{\cup}\left(\Gamma_{B} \tilde{\cup} \Gamma_{C}\right)$.

Bukti. Misal $\Gamma_{A}, \Gamma_{B}, \Gamma_{C} \in F S(U)$. Maka

(1) $\mu_{\gamma_{A}(x) \cup \gamma_{A}(x)}(u)=\max \left\{\mu_{\gamma_{A}(x)}(u), \mu_{\gamma_{A}(x)}(u)\right\}=\mu_{\gamma_{A}(x)}(u)$

(2) $\mu_{\gamma_{A}(x) \cup \gamma_{\phi}(x)}(u)=\max \left\{\mu_{\gamma_{A}(x)}(u), \mu_{\gamma_{\Phi}(x)}(u)\right\}$

$$
=\max \left\{\mu_{\gamma_{A}(x)}(u), 0\right\}=\mu_{\gamma_{A}(x)}(u)
$$

(3) $\mu_{\gamma_{A}(x) \cup \gamma_{\bar{E}}(x)}(u)=\max \left\{\mu_{\gamma_{A}(x)}(u), \mu_{\gamma_{\bar{E}}(x)}(u)\right\}$

$$
=1=\mu_{\gamma_{\bar{E}}(x)}(u)
$$

(4) $\mu_{\gamma_{A}(x) \cup \gamma_{B}(x)}(u)=\max \left\{\mu_{\gamma_{A}(x)}(u), \mu_{\gamma_{B}(x)}(u)\right\}$

$$
=\max \left\{\mu_{\gamma_{B}(x)}(u), \mu_{\gamma_{A}(x)}(u)\right\}
$$

(5) $\mu_{\left(\gamma_{A}(x) \cup \gamma_{B}(x)\right) \cup \gamma_{C}(x)}(u)=\max \left\{\max \left\{\mu_{\gamma_{A}(x)}(u), \mu_{\gamma_{B}(x)}(u)\right\}, \mu_{\gamma_{C}(x)}(u)\right\}$

$$
=\max \left\{\mu_{\gamma_{A}(x)}(u), \max \left\{\mu_{\gamma_{B}(x)}(u), \mu_{\gamma_{C}(x)}(u)\right\}\right\}
$$

Definisi 2.12. [2] Misal $\Gamma_{A}, \Gamma_{B} \in F S(U)$. Maka, irisan dari $\Gamma_{A}$ dan $\Gamma_{B}$, dinotasikan dengan $\Gamma_{A} \tilde{\cap} \Gamma_{B}$, didefinisikan oleh fungsi aproksimasi fuzzy

$$
\gamma_{A \tilde{\cap} B}(x)=\gamma_{A}(x) \cap \gamma_{B}(x) \text { untuk setiap } x \in E \text {. }
$$

Proposisi 2.13. [2] Misal $\Gamma_{A}, \Gamma_{B}, \Gamma_{C} \in F S(U)$. Maka,

(1) $\Gamma_{A} \tilde{\cap} \Gamma_{A}=\Gamma_{A}$.

(2) $\Gamma_{A} \tilde{\cap} \Gamma_{\Phi}=\Gamma_{\Phi}$.

(3) $\Gamma_{A} \tilde{\cap} \Gamma_{\bar{E}}=\Gamma_{A}$.

(4) $\Gamma_{A} \tilde{\cap} \Gamma_{B}=\Gamma_{B} \tilde{\cap} \Gamma_{A}$.

(5) $\left(\Gamma_{A} \tilde{\cap} \Gamma_{B}\right) \tilde{\cap} \Gamma_{C}=\Gamma_{A} \tilde{\cap}\left(\Gamma_{B} \tilde{\cap} \Gamma_{C}\right)$.

Bukti. Cara pembuktiannya mirip dengan bukti pada Proposisi 2.11.

Proposisi 2.14. [2] Misal $\Gamma_{A}, \Gamma_{B}, \Gamma_{C} \in F S(U)$. Maka, berlaku Hukum De Morgan sebagai berikut:

(1) $\left(\Gamma_{A} \tilde{\cup} \Gamma_{B}\right)^{\bar{c}}=\Gamma_{A}^{\bar{c}} \tilde{\cap} \Gamma_{B}^{\bar{c}}$.

(2) $\left(\Gamma_{A} \tilde{\cap} \Gamma_{B}\right)^{\bar{c}}=\Gamma_{A}^{\bar{c}} \tilde{\cup} \Gamma_{B}^{\bar{c}}$.

Bukti. Misal $\Gamma_{A}, \Gamma_{B}, \Gamma_{C} \in F S(U)$. 
(1) Perhatikan bahwa,

$$
\begin{aligned}
\gamma_{(A \tilde{\cup} B)^{\bar{c}}}(x) & =\gamma_{A \tilde{\cup} B}^{c}(x) \\
& =\left(\gamma_{A}(x) \cup \gamma_{B}(x)\right)^{c} \\
& =\gamma_{A}^{c}(x) \cap \gamma_{B}^{c}(x) \\
& =\gamma_{A^{\bar{c}}}(x) \cap \gamma_{B^{\bar{c}}}(x) \\
& =\gamma_{A^{\bar{c}} \tilde{\cap} B^{\bar{c}}}(x) .
\end{aligned}
$$

(2) Dibuktikan dengan cara yang sama.

Proposisi 2.15. [2] Misal $\Gamma_{A}, \Gamma_{B}, \Gamma_{C} \in F S(U)$. Maka,

(1) $\Gamma_{A} \tilde{\cup}\left(\Gamma_{B} \tilde{\cap} \Gamma_{C}\right)=\left(\Gamma_{A} \tilde{\cup} \Gamma_{B}\right) \tilde{\cap}\left(\Gamma_{A} \tilde{\cup} \Gamma_{C}\right)$.

(2) $\Gamma_{A} \tilde{\cap}\left(\Gamma_{B} \tilde{\cup} \Gamma_{C}\right)=\left(\Gamma_{A} \tilde{\cap} \Gamma_{B}\right) \tilde{\cup}\left(\Gamma_{A} \tilde{\cap} \Gamma_{C}\right)$.

Bukti. Misal $\Gamma_{A}, \Gamma_{B}, \Gamma_{C} \in F S(U)$.

(1) Perhatikan bahwa,

$$
\begin{aligned}
\gamma_{A \tilde{\cup}(B \tilde{\cap} C)}(x) & =\gamma_{A}(x) \cup \gamma_{(B \tilde{\cap} C)}(x) \\
& =\gamma_{A}(x) \cup\left(\gamma_{B}(x) \cap \gamma_{C}(x)\right) \\
& =\left(\gamma_{A}(x) \cup \gamma_{B}(x)\right) \cap\left(\gamma_{A}(x) \cup \gamma_{C}(x)\right) \\
& =\gamma_{A \tilde{\cup} B}(x) \cap \gamma_{A \tilde{\cup} C}(x) \\
& =\gamma_{(A \tilde{\cup} B) \tilde{\cap}(A \tilde{\cup} C)}(x) .
\end{aligned}
$$

(2) Dibuktikan dengan cara yang sama.

\section{Fuzzy Soft Aggregation}

Pada bagian ini, didefinisikan operator $f s$-aggregation yang menghasilkan kumpulan himpunan fuzzy dari $f s$-set dan himpunan kardinalnya.

Definisi 3.1. [2] Misal $\Gamma_{A} \in F S(U)$. Asumsikan bahwa $U=\left\{u_{1}, u_{2}, \cdots, u_{m}\right\}$, $E=\left\{x_{1}, x_{2}, \cdots, x_{n}\right\}$ dan $A \subseteq E$, maka $\Gamma_{A}$ dapat ditulis dalam bentuk tabel sebagai berikut

\begin{tabular}{c|c|c|c|c}
$\Gamma_{A}$ & $x_{1}$ & $x_{2}$ & $\cdots$ & $x_{n}$ \\
\hline$u_{1}$ & $\mu_{\gamma_{A}\left(x_{1}\right)}\left(u_{1}\right)$ & $\mu_{\gamma_{A}\left(x_{2}\right)}\left(u_{1}\right)$ & $\cdots$ & $\mu_{\gamma_{A}\left(x_{n}\right)}\left(u_{1}\right)$ \\
$u_{2}$ & $\mu_{\gamma_{A}\left(x_{1}\right)}\left(u_{2}\right)$ & $\mu_{\gamma_{A}\left(x_{2}\right)}\left(u_{2}\right)$ & $\ldots$ & $\mu_{\gamma_{A}\left(x_{n}\right)}\left(u_{2}\right)$ \\
$\vdots$ & $\vdots$ & $\vdots$ & $\ddots$ & $\vdots$ \\
$u_{m}$ & $\mu_{\gamma_{A}\left(x_{1}\right)}\left(u_{m}\right)$ & $\mu_{\gamma_{A}\left(x_{2}\right)}\left(u_{m}\right)$ & $\cdots$ & $\mu_{\gamma_{A}\left(x_{n}\right)}\left(u_{m}\right)$
\end{tabular}

dimana $\mu_{\gamma_{A}(x)}$ adalah fungsi keanggotaan dari suatu himpunan fuzzy atas $U$.

Jika $a_{i j}=\mu_{\gamma_{A}\left(x_{j}\right)}\left(u_{i}\right)$ untuk $i=1,2, \ldots$, m dan $j=1,2, \ldots, n$, maka fs-set $\Gamma_{A}$ secara tunggal dapat ditulis sebagai matriks 


$$
\left[a_{i j}\right]_{m \times n}=\left[\begin{array}{cccc}
a_{11} & a_{12} & \cdots & a_{1 n} \\
a_{21} & a_{22} & \cdots & a_{2 n} \\
\vdots & \vdots & \ddots & \vdots \\
a_{m 1} & a_{m 2} & \cdots & a_{m m}
\end{array}\right]
$$

disebut $m \times n$ fs-matrix dari fs-set $\Gamma_{A}$ atas $U$.

Definisi 3.2. [2] Misal $\Gamma_{A} \in F S(U)$. Maka himpunan kardinal dari $\Gamma_{A}$, dinotasikan dengan $c \Gamma_{A}$ dan didefinisikan oleh

$$
c \Gamma_{A}=\left\{\mu_{c \Gamma_{A}}(x) / x: x \in E\right\}
$$

adalah himpunan fuzzy atas E. Fungsi keanggotaan $\mu_{c \Gamma_{A}}$ dari $c \Gamma_{A}$ didefinisikan sebagai

$$
\mu_{c \Gamma_{A}}: E \rightarrow[0,1], \mu_{c \Gamma_{A}}(x)=\frac{\left|\gamma_{A}(x)\right|}{|U|}
$$

dimana $|U|$ adalah kardinalitas dari $U$, dan $\left|\gamma_{A}(x)\right|=\sum_{u \in U} \mu_{\gamma_{A}(x)}(u)$. Kumpulan semua himpunan-himpunan kardinal dari $f$ s-sets atas $U$ dinotasikan dengan $c F S(U)$. Jelas bahwa $c F S(U) \subseteq F(E)$.

Definisi 3.3. [2] Misal $\Gamma_{A} \in F S(U)$ dan $c \Gamma_{A} \in c F S(U)$. Asumsikan bahwa $E=$ $\left\{x_{1}, x_{2}, \ldots, x_{n}\right\}$ dan $A \subseteq E$, maka $c \Gamma_{A}$ dapat dituliskan dalam bentuk tabel sebagai berikut

\begin{tabular}{c|c|c|c|c}
$E$ & $x_{1}$ & $x_{2}$ & $\cdots$ & $x_{n}$ \\
\hline$\mu_{c \Gamma_{A}}$ & $\mu_{c \Gamma_{A}}\left(x_{1}\right)$ & $\mu_{c \Gamma_{A}}\left(x_{2}\right)$ & $\cdots$ & $\mu_{c \Gamma_{A}}\left(x_{n}\right)$
\end{tabular}

Jika $a_{1 j}=\mu_{c \Gamma_{A}}\left(x_{j}\right)$ untuk $j=1,2, \ldots, n$, maka $c \Gamma_{A}$ dapat dituliskan secara tunggal oleh matriks

$$
\left[a_{1 j}\right]_{1 \times n}=\left[\begin{array}{llll}
a_{11} & a_{12} & \cdots & a_{1 n}
\end{array}\right]
$$

yang disebut matriks kardinal dari himpunan kardinal $c \Gamma_{A}$ atas $E$.

Definisi 3.4. [2] Misal $\Gamma_{A} \in F S(U)$ dan $c \Gamma_{A} \in c F S(U)$. Maka, operator $f s$ aggregation, dinotasikan dengan $F S_{a g g}$, didefinisikan sebagai

$$
F S_{a g g}: c F S(U) \times F S(U) \rightarrow F(U), F S_{a g g}\left(c \Gamma_{A}, \Gamma_{A}\right)=A^{*}
$$

dimana

$$
A^{*}=\left\{\mu_{A^{*}}(u) / u: u \in U\right\}
$$

adalah himpunan fuzzy atas $U$. $A^{*}$ disebut agregat himpunan fuzzy dari $f$ s-set $\Gamma_{A}$. Fungsi keanggotaan $\mu_{A^{*}}$ dari $A^{*}$ didefinisikan sebagai berikut

$$
\mu_{A^{*}}: U \rightarrow[0,1], \mu_{A^{*}}(u)=\frac{1}{|E|} \sum_{x \in E} \mu_{c \Gamma_{A}(x)} \mu_{\gamma_{A}(x)}(u)
$$

dimana $|E|$ adalah kardinalitas dari $E$.

Definisi 3.5. [2] Misal $\Gamma_{A} \in F S(U)$ dan $A^{*}$ adalah agregat himpunan fuzzy. Asumsikan bahwa $U=\left\{u_{1}, u_{2}, \cdots, u_{m}\right\}$, maka $A^{*}$ dapat dituliskan dalam bentuk tabel sebagai berikut 


\begin{tabular}{c|c}
$\Gamma_{A}$ & $\mu_{A^{*}}$ \\
\hline$u_{1}$ & $\mu_{A^{*}}\left(u_{1}\right)$ \\
$u_{2}$ & $\mu_{A^{*}}\left(u_{2}\right)$ \\
$\vdots$ & $\vdots$ \\
$u_{m}$ & $\mu_{A^{*}}\left(u_{m}\right)$
\end{tabular}

Jika $a_{i 1}=\mu_{A^{*}}\left(u_{i}\right)$ untuk $i=1,2, \cdots, m$, maka $A^{*}$ secara tunggal dapat di-tuliskan

$$
\left[a_{i 1}\right]_{m \times 1}=\left[\begin{array}{c}
a_{11} \\
a_{21} \\
\vdots \\
a_{m 1}
\end{array}\right]
$$

disebut matriks agregat dari $A^{*}$ atas $U$.

Teorema 3.6. [2] Misal $\Gamma_{A} \in F S(U)$ dan $A \subseteq E$. Jika $M_{\Gamma_{A}}, M_{c \Gamma_{A}}$ dan $M_{A^{*}}$ adalah matriks representasi dari $\Gamma_{A}, c \Gamma_{A}$ dan $A^{*}$, maka

$$
|E| \times M_{A^{*}}=M_{\Gamma_{A}} \times M_{c \Gamma_{A}}^{T}
$$

dimana $M_{c \Gamma_{A}}^{T}$ adalah transpose dari $M_{c \Gamma_{A}}$ dan $|E|$ adalah kardinalitas dari $E$.

Bukti. Misal $\Gamma_{A} \in F S(U)$ dan $A \subseteq E$. Jika $M_{\Gamma_{A}}, M_{c \Gamma_{A}}$ dan $M_{A^{*}}$ adalah matriks representasi dari $\Gamma_{A}, c \Gamma_{A}$ dan $A^{*}$, maka akan ditunjukkan

$$
M_{A^{*}}=\frac{1}{|E|} \times M_{\Gamma_{A}} \times M_{c \Gamma_{A}}^{T} .
$$

Perhatikan bahwa:

$$
\begin{aligned}
{\left[\begin{array}{c}
\mu_{A^{*}}\left(u_{1}\right) \\
\mu_{A^{*}}\left(u_{2}\right) \\
\vdots \\
\mu_{A^{*}}\left(u_{m}\right)
\end{array}\right]=\frac{1}{|E|}\left[\begin{array}{c}
\sum_{i=1}^{n} \mu_{\gamma_{A}\left(x_{i}\right)}\left(u_{1}\right) \mu_{c \Gamma_{A}}\left(x_{i}\right) \\
\sum_{i=1}^{n} \mu_{\gamma_{A}\left(x_{i}\right)}\left(u_{2}\right) \mu_{c \Gamma_{A}}\left(x_{i}\right) \\
\vdots \\
\sum_{i=1}^{n} \mu_{\gamma_{A}\left(x_{i}\right)}\left(u_{m}\right) \mu_{c \Gamma_{A}}\left(x_{i}\right)
\end{array}\right] } \\
=\frac{1}{|E|}\left[\begin{array}{cccc}
\mu_{\gamma_{A}\left(x_{1}\right)}\left(u_{1}\right) & \mu_{\gamma_{A}\left(x_{2}\right)}\left(u_{1}\right) & \cdots & \mu_{\gamma_{A}\left(x_{n}\right)}\left(u_{1}\right) \\
\mu_{\gamma_{A}\left(x_{1}\right)}\left(u_{2}\right) & \mu_{\gamma_{A}\left(x_{2}\right)}\left(u_{2}\right) & \cdots & \mu_{\gamma_{A}\left(x_{n}\right)}\left(u_{2}\right) \\
\vdots & \vdots & \ddots & \vdots \\
\mu_{\gamma_{A}\left(x_{1}\right)}\left(u_{m}\right) & \mu_{\gamma_{A}\left(x_{2}\right)}\left(u_{m}\right) & \cdots & \mu_{\gamma_{A}\left(x_{n}\right)}\left(u_{m}\right)
\end{array}\right]\left[\begin{array}{c}
\mu_{c \Gamma_{A}}\left(x_{1}\right) \\
\mu_{c \Gamma_{A}}\left(x_{2}\right) \\
\vdots \\
\mu_{c \Gamma_{A}}\left(x_{n}\right)
\end{array}\right]
\end{aligned}
$$

\section{Aplikasi Fuzzy Soft Set Pada Penerimaan Pekerja}

PT. Mulia Boga Raya yang dikenal dengan produksi keju Prochiz telah membuka lowongan untuk posisi Manager Engineering dengan parameter-parameter yang dipertimbangkan antara lain: Pria, Pendidikan Minimal S1 Teknik Elektro, Teknik Mesin/ Automasi Industri, Usia Produktif (Dalam Pekerjaan) Antara 35 45, Sehat Jasmani dan Rohani, Domisili Jabodetabek, Lancar Berbahasa Inggris, Pengalaman sebagai Manager atau sebagai Asisten Manager, Paham Tentang Programmable Logic Control, Paham Tentang Total Preventive Maintanance, Paham 
Tentang ISO 22000, Menguasai Aplikasi-aplikasi Komputer (Microsoft Word, Office, Power Point, Corel Draw, dan Auto Cad).

Berdasarkan persyaratan-persyaratan di atas, dapat ditulis himpunan parameter $E=\left\{x_{1}, x_{2}, x_{3}, x_{4}, \ldots, x_{11}\right\}$. Dipilih beberapa persyaratan yang sangat dipertimbangkan, yaitu $A=\left\{x_{3}, x_{4}, x_{6}, x_{7}, x_{8}, x_{9}, x_{10}, x_{11}\right\}$. Setelah seleksi administrasi, ada 20 orang kandidat yang memenuhi persyaratan tersebut yang disimbolkan dengan $u_{i}$, untuk $i=1,2, \ldots, 20$. Misalkan himpunan $U=\left\{u_{1}, u_{2}, u_{3}, \ldots, u_{20}\right\}$. Kemudian, untuk mencari calon terbaik dijalankan algoritma sebagai berikut :

Langkah 1: Bentuk $f_{s-\text { set }} \Gamma_{A}$ atas $U$.

$\Gamma_{A}=\left\{\left(x_{3},\left\{0.9 / u_{1}, 0.9 / u_{2}, 1 / u_{3}, 1 / u_{4}, 0.9 / u_{5}, 0.9 / u_{6}, 0.9 / u_{7}, 0.9 / u_{8}, 0.8 / u_{9}, 0.8 / u_{10}\right.\right.\right.$, $\left.\left.0.9 / u_{11}, 0.8 / u_{12}, 0.8 / u_{13}, 1 / u_{14}, 0.8 / u_{15}, 1 / u_{16}, 1 / u_{17}, 1 / u_{18}, 0.8 / u_{19}, 0.9 / u_{20}\right\}\right)$, $\left(x_{4},\left\{0.9 / u_{1}, 0.9 / u_{2}, 0.9 / u_{3}, 0.85 / u_{4}, 0.9 / u_{5}, 0.9 / u_{6}, 0.9 / u_{7}, 0.9 / u_{8}, 0.9 / u_{9}, 0.9 / u_{10}\right.\right.$, $0.8 / u_{11}, 0.9 / u_{12}, 0.9 / u_{13}, 0.9 / u_{14}, 0.75 / u_{15}, 0.9 / u_{16}, 0.85 / u_{17}, 0.9 / u_{18}, 0.9 / u_{19}$, $\left.\left.0.9 / u_{20}\right\}\right),\left(x_{6},\left\{0.85 / u_{1}, 0.85 / u_{2}, 0.7 / u_{3}, 0.7 / u_{4}, 0.7 / u_{5}, 0.85 / u_{6}, 0.8 / u_{7}\right.\right.$, $0.7 / u_{8}, 0.7 / u_{9}, 0.75 / u_{10}, 0.7 / u_{11}, 0.7 / u_{12}, 0.65 / u_{13}, 0.6 / u_{14}, 0.7 / u_{15}, 0.65 / u_{16}$, $\left.\left.0.6 / u_{17}, 0.75 / u_{18}, 0.85 / u_{19}, 0.7 / u_{20}\right\}\right),\left(x_{7},\left\{1 / u_{1}, 0.9 / u_{2}, 0.9 / u_{3}, 0.9 / u_{4}, 0.9 / u_{5}\right.\right.$, $0.8 / u_{6}, 1 / u_{7}, 0.8 / u_{8}, 0.9 / u_{9}, 0.9 / u_{10}, 0.8 / u_{11}, 0.8 / u_{12}, 0.8 /{ }_{13}, 0.8 / u_{14}, 1 / u_{15}$, $\left.\left.0.9 / u_{16}, 0.9 / u_{17}, 1 / u_{18}, 0.9 / u_{19}, 0.9 / u_{20}\right\}\right),\left(x_{8},\left\{0.8 / u_{1}, 0.8 / u_{2}, 0.85 / u_{3}, 0.8 / u_{4}\right.\right.$, $0.75 / u_{5}, 0.8 / u_{6}, 0.85 / u_{7}, 0.85 / u_{8}, 0.75 / u_{9}, 0.85 / u_{10}, 0.8 / u_{11}, 0.85 / u_{12}, 0.8 / u_{13}$, $\left.\left.0.8 / u_{14}, 0.85 / u_{15}, 0.8 / u_{16}, 0.8 / u_{17}, 0.8 / u_{18}, 0.85 / u_{19}, 0.8 / u_{20}\right\}\right),\left(x_{9},\left\{0.9 / u_{1}\right.\right.$, $0.9 / u_{2}, 0.8 / u_{3}, 0.75 / u_{4}, 0.8 / u_{5}, 0.85 / u_{6}, 0.85 / u_{7}, 0.75 / u_{8}, 0.8 / u_{9}, 0.75 / u_{10}, 0.85 / u_{11}$, $\left.\left.0.8 / u_{12}, 0.8 / u_{13}, 0.75 / u_{14}, 0.7 / u_{15}, 0.7 / u_{16}, 0.8 / u_{17}, 0.85 / u_{18}, 0.9 / u_{19}, 0.75 / u_{20}\right\}\right)$, $\left(x_{10},\left\{0.9 / u_{1}, 0.75 / u_{2}, 0.75 / u_{3}, 0.8 / u_{4}, 0.75 / u_{5}, 0.75 / u_{6}, 0.8 / u_{7}, 0.7 / u_{8}, 0.75 / u_{9}\right.\right.$, $0.8 / u_{10}, 0.75 / u_{11}, 0.7 / u_{12}, 0.75 / u_{13}, 0.75 / u_{14}, 0.8 / u_{15}, 0.8 / u_{16}, 0.75 / u_{17}, 0.75 / u_{18}$, $\left.\left.0.8 / u_{19}, 0.8 / u_{20}\right\}\right),\left(x_{11},\left\{0.9 / u_{1}, 0.9 / u_{2}, 0.8 / u_{3}, 0.85 / u_{4}, 0.9 / u_{5}, 0.9 / u_{6}, 0.9 / u_{7}\right.\right.$, $0.9 / u_{8}, 0.8 / u_{9}, 0.9 / u_{10}, 0.85 / u_{11}, 0.85 / u_{12}, 0.9 / u_{13}, 0.85 / u_{14}, 0.85 / u_{15}, 0.8 / u_{16}$, $\left.\left.\left.0.8 / u_{17}, 0.8 / u_{18}, 0.9 / u_{19}, 0.8 / u_{20}\right\}\right)\right\}$.

Langkah 2: Hitung himpunan kardinal dari $\Gamma_{A}$ untuk setiap $x \in E$. $c \Gamma_{A}=\left\{0.9 / x_{3}, 0.8825 / x_{4}, 0.725 / x_{6}, 0.89 / x_{7}, 0.8125 / x_{8}, 0.8025 / x_{9}, 0.77 / x_{10}, 0.8575 / x_{11}\right\}$

Langkah 3: Hitung aggregate himpunan fuzzy menggunakan teorema (1) Sehingga diperoleh $A^{*}=\left\{0.54068 / u_{1}, 0.52209 / u_{2}, 0.50899 / u_{3}, 0.50503 / u_{4}, 0.50122 / u_{5}, 0.51035 / u_{6}\right.$, $0.53043 / u_{7}, 0.49336 / u_{8}, 0.48524 / u_{9}, 0.50337 / u_{10}, 0.48855 / u_{11}, 0.48493 / u_{12}$, $0.48534 / u_{13}, 0.49086 / u_{14}, 0.48878 / u_{15}, 0.4982 / u_{16}, 0.49469 / u_{17}, 0.52033 / u_{18}$, $\left.0.5211 / u_{19}, 0.49697 / u_{20}\right\}$.

Langkah 4: Pilih nilai keanggotaan yang terbesar, yaitu:

$$
\max \mu_{A^{*}}(u)=0.54068
$$

yang berarti pelamar $u_{1}$ memungkinkan diterima untuk pekerjaan ini. 


\section{Ucapan Terima kasih}

Penulis mengucapkan terima kasih kepada Bapak Dr. Admi Nazra, Dr. Mahdhivan Syafwan, Ibu Nova Noliza Bakar, M.Si, Ibu Monika Rianti Helmi, M.Si, dan Ibu Dr. Lyra Yulianti yang telah memberikan masukan dan saran sehingga paper ini dapat diselesaikan dengan baik.

\section{Daftar Pustaka}

[1] Aktas, H., and N. Cagman. 2007. Soft set and soft group. Information Sciences. 117: $2726-2735$

[2] Cagman, N., S. Enginoglu and F. Citak. 2011. Fuzzy soft set theory and its application. Iranian Journal of Fuzzy System. 8(3): 137 - 147

[3] Jantzen, J. 1998. Tutorial On Fuzzy Logic. citeseerx.ist.psu.edu, diakses tanggal 18 Maret 2015

[4] Maji, P. K., R. Biswas, and A. R. Roy. 2003. Soft set theory. Comput. Math. Appl. 45:555 - 562

[5] Molodtsov, D. A. 1999. Soft set theory-first result. Computers and Mathematics with Applications. (37): $19-31$

[6] Roy, A. R., and P. K. Maji. 2007. A fuzzy soft set theoretic approach and decision making problems. J. Comput. Appl. Math. 203:412-418

[7] Zadeh, L.A. 1965. Fuzzy sets. Information and Control. 8: 338 - 353 